\title{
Novel Alternative Treatment to Plasmapheresis in Evans Syndrome
}

\author{
Martin-Lazaro $\mathrm{JF}^{* 1}$, Naganathar $\mathrm{S}^{1}$, $\mathrm{d}^{\prime}$ Espiney $\mathrm{R}^{2}$, Brys $\mathrm{M}^{2}$, Norkiene $\mathrm{I}^{2}$, Grundlingh $\mathrm{J}^{2}$, Kreze $\mathrm{O}^{2}$ and \\ Napier $\mathbf{J}^{1}$
}

${ }^{1}$ Intensive Care Unit, Newham University Hospital, United Kingdom

${ }^{2}$ Haematology Department, Newham University Hospital, United Kingdom

${ }^{*}$ Corresponding author: Martin-Lazaro JF, Intensive Care Unit, Newham University Hospital, United Kingdom, E-mail: juanfranciscoml@gmail.com

Citation: Martin-Lazaro JF, Naganathar S, d'Espiney R, Brys M, Norkiene I, et al. (2018) Novel Alternative Treatment To Plasmapheresis In Evans Syndrome. J Hematol Blood Disord 4(1): 102.

doi: 10.15744/2455-7641.4.102

Received Date: March 30, 2018 Accepted Date: August 14, 2018 Published Date: August 16, 2018

\begin{abstract}
Evans syndrome is a rare haematological disease characterised by concurrent occurrence of autoimmune haemolytic anaemia (AIHA) and idiopathic thrombocytopenic purpura. The autoimmune haemolysis can lead to hyperbilirubinaemia, profound anaemia and haemodynamic instability. It can be difficult to treat and when refractory to medical treatment it could necessitate emergency splenectomy. Plasmapheresis is used in refractory haemolytic anaemia to aid removal of disease causing autoimmune complexes whilst immunomodulators act to reduce autoantibody response and effectiveness. However, this is an expensive and resource intensive process that is limited to tertiary referral centres. In this case study we present a successful novel alternative for life threatening AIHA in Evans Syndrome using haemofiltration with on-going fresh frozen plasma transfusion when resources are limited or it is not an accessible method.
\end{abstract}

Keywords: Autoimmune Haemolytic Anaemia; Evans syndrome; Plasmapheresis

\section{Introduction}

Autoimmune haemolytic anaemia (AIHA) is often an IgG driven condition, which is a haematological emergency requiring immunomodulation. Rarely, AIHA occurs concurrently with idiopathic thrombocytopenic purpura. The condition that combines AIHA and immune thrombocytopenia has been denominated Evans syndrome, which is more difficult to treat and has a higher mortality than AIHA presenting alone. This condition can be primary idiopathic or secondary to diverse factors: viral infections, autoimmune and connective tissue diseases, immune deficiency diseases, malignancies, babesiosis after splenectomy or hematopoietic disorders [1]. Antibody in AIHA destroys the red cells primarily by binding to Fc receptors on effector cells (phagocytes) of the immune system; binding results also in phagocytosis (partial or complete) or membrane rupture which this occurs ultimately in the spleen. AIHA symptoms are determined by the severity of the anaemia in which intravascular red cell destruction leads to an insufficient oxygenation, cardiac failure and rise in serum bilirubin, becoming a life - threatening condition without treatment [2]. First-line therapy for AIHA remains high dose prednisolone (1-2mg/kg/day), with Rituximab (RXB) as second line therapy due to limited evidence on its long term benefits [3-5]. In patients failing medical management, plasmapheresis aids the removal of pathogenic immune complexes [6,7]. However, this procedure is labour-intensive, costly and limited to specialist centres. In this case report we describe an alternative method when there is no response to medical treatment, using a conventional veno-venous haemofiltration with plasma to aid the potential removal of IgG immune complexes.

\section{Objectives}

To describe the use of conventional veno-venous haemofiltration $(\mathrm{CVVH})$ with plasma in Evans syndrome as a bridging treatment in life - threatening autoimmune haemolytic anaemia.

\section{Methods}

High volume veno-venous hemofiltration was performed using the Aquarius System, in which blood was driven through a highly permeable hemofilter type Aquamax HF 12 poly-ether-sulfone from Baxter (cut-off point of $30 \mathrm{kDa}$ ). Epoprostenol as an anticoagulant due to concomitant thrombocytopenia was added to the filter. Extracorporeal blood flow ranged from 250 to 360 $\mathrm{ml} / \mathrm{min}(60 \mathrm{ml} / \mathrm{Kg})$. Pre dilution mode was used. The principle of clearance in this model of continuous dialysis was convection. Even to negative daily balance was achieved with minimal dose of vasopressors (noradrenaline $0.2 \mathrm{mcg} / \mathrm{kg} / \mathrm{min}$ ) to maintain 
haemodynamic stability. The procedure was performed over 72 hours. Fresh frozen plasma ( 2 units, 6 hourly; approximately 250 $\mathrm{ml}$ per unit), was administered during the procedure. One filter clotted and had to be changed in the first $24 \mathrm{~h}$ of the procedure. Two units of packed red blood cells (PRBC) were transfused during the procedure and one pool of platelets administered as well, due to severe thrombocytopenia, possibly in relation of mechanical rupture by the hemofiltration and his concomitant ITP. No other complications were observed during the procedure.

Informed consent was obtained from the family who was informed of the novel nature of the therapy and warned that it may be halted if any complications arose or if no improvement was seen.

The patient's blood was processed in the hospital laboratory prior to starting the procedure and $12 \mathrm{~h}$ hourly after the initiation of hemofiltration. Metabolic state was monitored through arterial blood gas each 2 hours.

\section{Case Report}

A 54-year-old man with known idiopathic thrombocytopenia purpura (IT) on long term prednisolone presented to the emergency department following a collapse. In the preceding two days he had been experiencing melena, postural syncope, shortness of breath and lethargy. This was all preceded by flu-like illness in the week before. He was admitted to hospital with a rapid deterioration. Suspecting an association with AIHA he was admitted to the intensive care unit (ICU) with high dose Prednisolone (60mg once a day), intravenous immunoglobulin (IVIG) and packed red blood cell (PRBC) transfusion to maintain haemodynamic stability without a specific haemoglobin ( $\mathrm{Hb}$ ) target due to profound haemolysis despite administration of 12 PRBC.

On admission to ICU he was tachycardic (110 beats per min), hypoxic (O2 Sat $85 \%$ on room air) and pyrexial ( $\left.37.8^{\circ} \mathrm{C}\right)$. The patient was lethargic with a Glasgow coma score of 9, jaundiced with encephalopathy grade II. Signs of cardiac failure were present with bibasal pulmonary crepitates, jugular ingurgitation, hepatomegaly and bilateral limb oedema. Abdomen was soft but with marked hepato - splenomegaly. Coluric urinary output was preserved.

Laboratory parameters on ICU admission were: sodium $139 \mathrm{mmol} / \mathrm{L}$, potassium $3.9 \mathrm{mmol} / \mathrm{L}$, chloride $105 \mathrm{mmol} / \mathrm{L}$, urea 10.3 $\mathrm{mmo} / \mathrm{L}$ and creatinine $85 \mathrm{umol} / \mathrm{L}$; haemoglobin $(\mathrm{Hb}) 71 \mathrm{~g} / \mathrm{L}$, white cell count (WCC) $8.9 \times 10^{9} / \mathrm{L}$, platelet count $70 \times 10^{9} / \mathrm{L}$, bilirubin 1067 umol/L, LDH $1911 \mathrm{U} / \mathrm{L}$, alkaline phosphatase (ALP) $57 \mathrm{U} / \mathrm{L}$, alanine transaminase (ALT) $33 \mathrm{U} / \mathrm{L}$, albumin 48g/L and normal coagulation (PT 10.9 seconds, INR 1.1, APTT 32 seconds and APTTr 1.2).

To achieve immune - suppression he was also started on mycophenolate mofetil (MMF), $1 \mathrm{~g}$ twice a day, and RXB. Empirically was started on meropenem as he was persistently pyrexial with no obvious source with CRP $9 \mathrm{mg} / \mathrm{dl}$ and negative procalcitonin. Despite optimal medical management, his haemolysis continued, therefore, he was referred to our tertiary haematology centre for plasmapheresis. However, due to a lack of beds and instability of the patient, it was decided to be managed locally in the interim. As a compassionate alternative we initiated high volume CVVH with plasma as described in the methods, which resulted in a rapid clinical improvement and dramatically decrease of transfusion requirements. On discharge, cyclosporine $50 \mathrm{mg}$ twice a day and Danazol 100 mg daily was started.

To complete the study, we performed a bone marrow biopsy which revealed a hypocellular marrow without abnormal cellularity. IgG antibodies and direct antiglobulin (Coombs) was positive with decreased haptoglobin levels. CT abdomen showed a $21 \mathrm{~mm}$ polypoid looking lesion in the terminal ileum which biopsy discarded a malignancy; his blood cultures during his admission, respiratory virus, HIV serology and hepatitis screening were negative.

\section{Results}

Clinical improvement was observed after $24 \mathrm{~h}$ of plasma - CVVH with normalisation of heart rate, respiratory rate, oxygenation (no endotracheal intubation was required but supported with nasal - high flow oxygen therapy: FiO2 40\% and 40L of flow) and disappearance of cardiac failure signs.

Guidance of therapy effectiveness was controlled clinically and through laboratory parameters: LDH and bilirubin which were corrected in around 80\% of its ICU admission levels on discharge (LDH 79.37\% and bilirubin 74.49\%).

Laboratory progress can be observed in the following Figure 1 and Table 1:

\begin{tabular}{|c|c|c|c|c|c|c|c|c|c|c|c|}
\hline \multirow[t]{2}{*}{ TIME } & \multirow[t]{2}{*}{ ADMISSION } & \multicolumn{10}{|c|}{ ICU } \\
\hline & & Day 1 & Day 2 & $\begin{array}{c}\text { Day } 3 \\
\text { am }\end{array}$ & $\begin{array}{c}\text { Day } 3 \\
\text { pm }\end{array}$ & $\begin{array}{c}\text { Day } 4 \\
\text { am }\end{array}$ & $\begin{array}{c}\text { Day } 4 \\
\text { pm }\end{array}$ & $\begin{array}{c}\text { Day } 5 \\
\text { am }\end{array}$ & $\begin{array}{c}\text { Day } 5 \\
\text { pm }\end{array}$ & Day 6 & Discharge \\
\hline TREATMENT: & $\begin{array}{l}\text { Steroids / } \\
\text { IVIG }\end{array}$ & MMF & RXB & \multicolumn{5}{|c|}{ PLASMA - CVVH } & & & \\
\hline LDH (U/L) & 2847 & 1911 & & 3126 & & & & & 2052 & & 645 \\
\hline Bilirubin(umol/L) & 490 & 384 & 847 & 1067 & 898 & 878 & 898 & 468 & 699 & 372 & 252 \\
\hline WCC $\left(10^{*} 9 / \mathrm{L}\right)$ & 13.3 & 8.9 & 11.7 & 13.5 & 12 & 8 & 9.7 & 4.1 & 5 & 5 & 8 \\
\hline $\mathrm{Hb}(\mathrm{g} / \mathrm{L})$ & 45 & 71 & 59 & 79 & 66 & 86 & 93 & 92 & 76 & 77 & 105 \\
\hline Platelets $(10 * 9 / \mathrm{L})$ & 216 & 70 & 100 & 100 & 93 & 67 & 79 & 29 & 51 & 50 & 187 \\
\hline
\end{tabular}




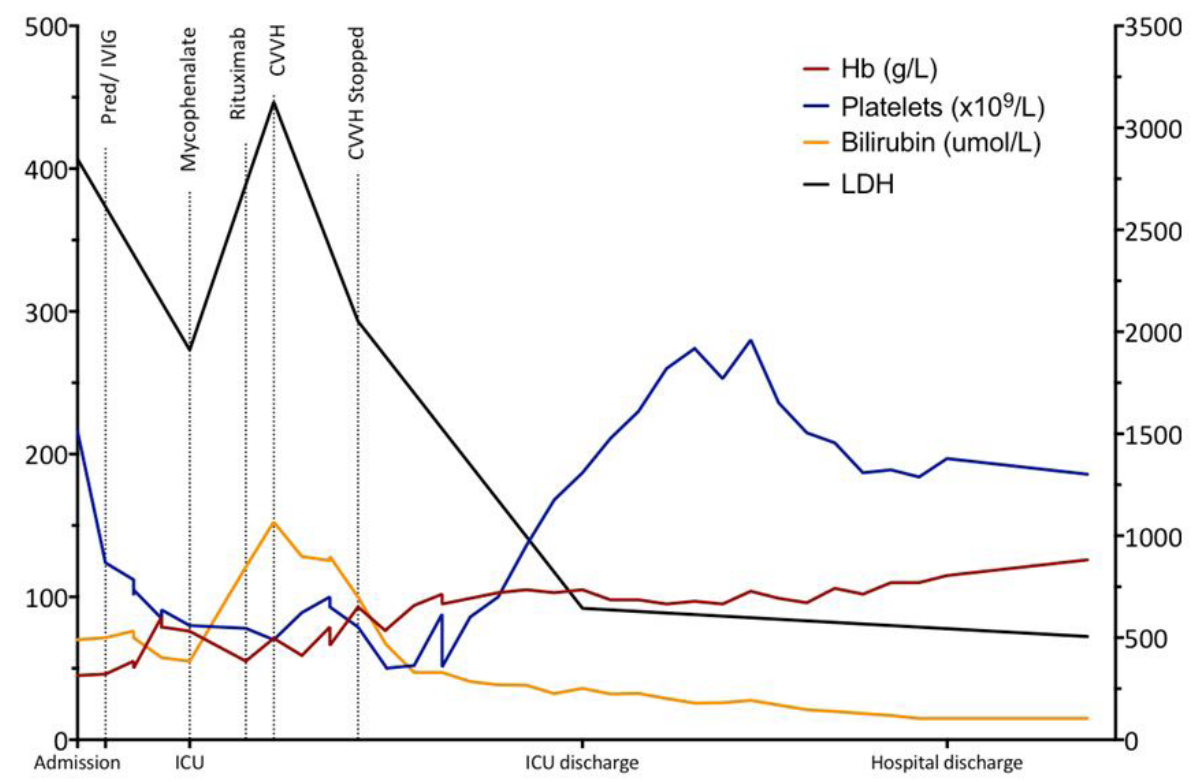

Figure 1: The progression of serum levels of haemoglobin $(\mathrm{g} / \mathrm{L})$ and platelets $(\mathrm{x} 109 / \mathrm{L})$ on the left axis and bilirubin (umol/L) on the $\mathrm{LDH}(\mathrm{U} / \mathrm{L})$ on the right axis to illustrate the change in biochemical indicators of disease progression with medical interventions

\section{Discussion}

The prognosis of ITP can be determined when associated with severe AIHA, whether the rapidity with which the anaemia develops and whether there is concurrent illness. In healthy patients the limits for compensation and optimal oxygen delivery can be maintained at a haemoglobin concentration as low as $5 \mathrm{~g} / \mathrm{dl}$ (equivalent to a haematocrit of 15\%) [8]. Evans syndrome may become life - threatening if haemoglobin falls below a level able to sustain sufficient oxygenation, cardiac decompensation occurs and is accompanied of severe hyperbilirubinaemia in which the patient could present low level of consciousness. In such patients, corrective measures must be taken at once (glucocorticoids, immunosuppressors, intravenous immune globulin, transfusion or plasmapheresis). Although immunosuppression is the recommended treatment, plasmapheresis is utilised to immunomodulate the patients while the medical treatment is optimised [9]. In our patient, access to plasmapheresis was hindered due to lack of bed availability at the tertiary centre, which is not an unfamiliar scenario when resources are limited. Under this setting we looked for a simple compassionate alternative that could be conducted using conventional renal replacement therapy by simply administering to the patient fresh frozen plasma. The results were clinically evident in 24 hours, in parallel with the normalisation of laboratory parameters which turned into a successful outcome for our patient.

\section{Conclusion}

Plasma - CVVH was a safe and efficient alternative in life - threatening AIHA associated with ITP when there is no response to medical treatment. This therapeutic method available in most of intensive care units is less labour, cost and resource intensive than plasmapheresis and, can halt further deterioration in critically ill patients in the face of limited resources.

\section{References}

1. Michel M, Chanet V, Dechartres A, Morin AS, Piette JC, et al. (2009) The spectrum of Evans syndrome in adults: new insight into the disease based on the analysis of 68 cases. Blood 114: 3167-72.

2. Gehrs BC, Friedberg RC (2002) Autoimmune hemolytic anemia. Am J Hematol 69: 258-71.

3. Crowther M, Chan YL, Garbett IK, Lim W, Vickers MA, et al. (2011) Evidence-based focused review of the treatment of idiopathic warm immune hemolytic anemia in adults. Blood 118: 4036-40.

4. Pescovitz MD, Torgerson TR, Ochs HD, Ocheltree E, McGee P, et al. (2011) Effect of rituximab on human in vivo antibody immune responses. J Allergy Clin Immunol 128: 1295-302.

5. Reynaud Q, Durieu I, Dutertre M, Ledochowski S, Durupt S, et al. (2015) Efficacy and safety of rituximab in auto-immune hemolytic anemia: A meta-analysis of 21 studies. Autoimmun Rev 14: 304-13.

6. Silberstein LE, Berkman EM (1983) Plasma exchange in autoimmune hemolytic anemia (AIHA). J Clin Apher 1: 238-42.

7. Schwartz J, Winters JL, Padmanabhan A, Balogun RA, Delaney M, et al. (2013) Guidelines on the Use of Therapeutic Apheresis in Clinical Practice-EvidenceBased Approach from the Writing Committee of the American Society for Apheresis: The Sixth Special Issue. Shaz B, editor. J Clin Apheresis 28: 145-284.

8. Weiskopf RB, Viele MK, Feiner J, Kelley S, Lieberman J, et al. (1998) Human cardiovascular and metabolic response to acute, severe isovolemic anemia. JAMA 279: $217-21$.

9. Lechner K, Jager U (2010) How I treat autoimmune hemolytic anemias in adults. Blood 116: 1831-8. 


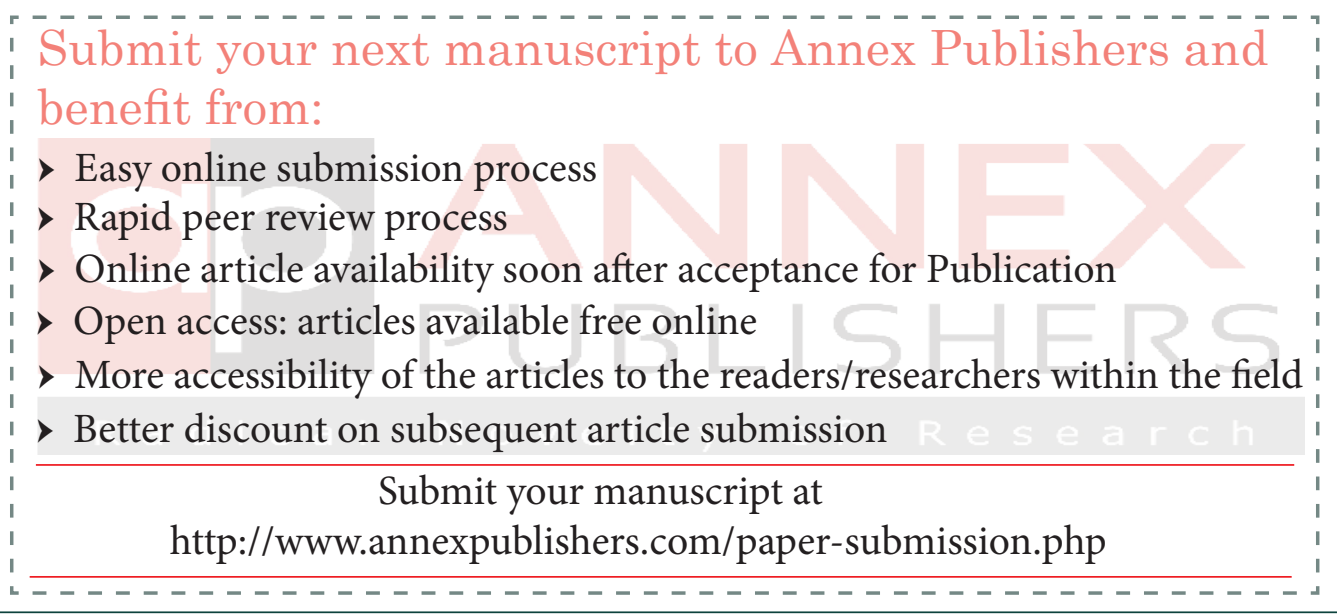

